\title{
Maximizing the Tweet Engagement Rate in Academia: Analysis of the AJNR Twitter Feed
}

\author{
(D) V. Wadhwa, (DE. Latimer, (D). Chatterjee, DJ. McCarty, and (DR.T. Fitzgerald
}

\section{ABSTRACT}

SUMMARY: The use of social media by medical professionals and organizations is increasing, with Twitter receiving the most attention. User engagement is an important goal of social media activity, and engagement metrics represent a viable gauge of value in social media. No thorough analysis of tweet characteristics that increase academic user engagement has yet been published. In this study, the authors analyzed the American Journal of Neuroradiology Twitter feed to determine the tweet characteristics that were associated with higher engagement rates.

T witter (Twitter Inc, San Francisco, California), which is primarily based on status updates ("tweets") consisting of 140 characters or fewer, with an optional 6 pictures or fewer, is one of the largest social media platforms. Twitter is increasingly being adopted by radiologists, as is evident at various national meetings. ${ }^{1}$ Many of the widely circulated general and subspecialty radiology journals, including Radiology, RadioGraphics, and the American Journal of Neuroradiology (AJNR), use social media to increase readership and interact with readers and authors, providing a dynamic platform for scientific discussion. ${ }^{2}$ User engagement is the key outcome of tweeting for individual users and organizations alike, and irrespective of content, some tweets may be more engaging than others. However, the tweet characteristics associated with a high engagement rate have not yet been studied for radiology journals. The objective of this study was to identify the characteristics of AJNR tweets associated with high user engagement rate.

\section{MATERIALS AND METHODS \\ Data Source}

The AJNR Twitter account (@TheAJNR) has been active since January 2013 and has more than 4000 tweets and 2500 followers as of February 2017. Twitter analytics, which include impressions,

Received April 6, 2017; accepted after revision May 4.

From the Departments of Radiology (V.W., E.L., R.T.F.) and Internal Medicine (K.C.), University of Arkansas for Medical Sciences, Little Rock, Arkansas; and Department of Radiology and Imaging Sciences (J.M.), Emory University, Atlanta, Georgia.

Please address correspondence to Vibhor Wadhwa, MD, Department of Radiology, University of Arkansas for Medical Sciences, 4301 W Markham St Little Rock, AR 72205; e-mail: vibhorwadhwa90@gmail.com; @wadhwa_rad

三 Indicates article with supplemental on-line appendix.

http://dx.doi.org/10.3174/ajnr.A5283 engagements, and engagement rate for each tweet, were obtained from the AJNR Twitter account for tweets published from August 2015-July 2016. Impressions refer the number of times a Twitter user is served a tweet within their timeline or as part of a search result. Engagements are defined by the total number of times a user interacted with a tweet. Clicks anywhere on the tweet, including retweets, replies, follows, likes, links, cards, hashtags, embedded media, username, profile photo, or tweet expansion, result in engagement. The engagement score for each tweet was calculated as the number of engagements divided by impressions.

\section{Tweet Characteristics}

For this study, tweets with an engagement score above the 75th percentile were considered as "high engagement tweets," and tweets below the 25th percentile were considered as "low engagement tweets." Each tweet was further characterized by the month, time of day (morning, afternoon, evening, and night), weekend versus weekday, presence or absence of an imbedded image or hashtag, and type of tweet. The time of day in Central Standard Time (CST) was divided as morning (06:00-11:59 hours), afternoon (12:00-16:59 hours), evening (17:00-20:59 hours) and night (21:00-05:59 hours). The type of tweet was divided into the following subtypes: Fellows' Journal Club, Editor's Choice Article, Tweet Chat, Case, Article, Blog, and Miscellaneous.

\section{Statistical Analysis}

All data were stored in Excel 2013 (Microsoft, Redmond, Washington) and analyzed by using SPSS version 23 (IBM, Armonk, New York). A multivariate logistic regression model was constructed to identify the tweet characteristics associated with a high engagement rate. A 2 -sided $P$ value $<.05$ was considered statistically significant. 
Table 1: Frequency distribution of tweet characteristics

\begin{tabular}{|c|c|c|c|c|c|c|}
\hline Characteristic & Frequency & $\begin{array}{l}\text { High ER } \\
\text { Tweets }\end{array}$ & \% High ER & $\begin{array}{l}\text { Odds } \\
\text { Ratio }^{a}\end{array}$ & $95 \% \mathrm{Cl}^{\mathrm{a}}$ & $P$ Value \\
\hline \multicolumn{7}{|l|}{ Picture } \\
\hline Yes & 405 & 235 & $58.02 \%$ & 28.749 & $17.654-46.816$ & .000 \\
\hline No & 627 & 23 & $3.67 \%$ & $\#$ & & \\
\hline \multicolumn{7}{|l|}{ Hashtag } \\
\hline Yes & 751 & 242 & $32.22 \%$ & 3.266 & $1.747-6.108$ & .000 \\
\hline No & 281 & 16 & $5.69 \%$ & $\#$ & & \\
\hline Weekday & 910 & 231 & $25.38 \%$ & 0.613 & $0.348-1.081$ & .91 \\
\hline Weekend & 122 & 27 & $22.13 \%$ & $\#$ & & \\
\hline \multicolumn{7}{|l|}{ Time of day } \\
\hline Morning & 606 & 200 & $33.00 \%$ & $\#$ & $0.298-0.730$ & .001 \\
\hline Afternoon & 318 & 47 & $14.78 \%$ & 0.467 & $0.249-1.842$ & .445 \\
\hline Evening & 66 & 7 & $10.60 \%$ & 0.677 & $0.115-1.773$ & .254 \\
\hline Night & 42 & 4 & $9.52 \%$ & 0.452 & & \\
\hline
\end{tabular}

Note:- \# indicates reference category; ER, engagement rate.

a From the logistic regression model. The "Month of Tweet" (Fig 1) was also part of the logistic model and did not show any independent statistical significance.

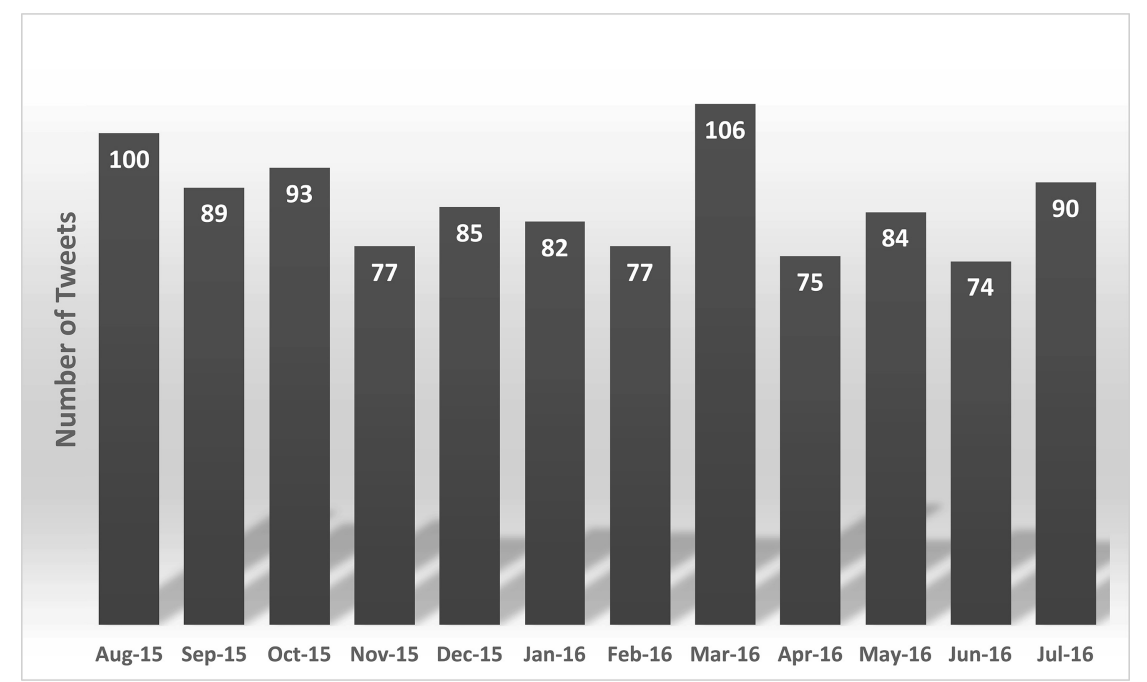

FIG 1. Tweet frequency per month over the study period.

Table 2: Distribution by tweet types

\begin{tabular}{lc}
\hline \multicolumn{1}{c}{ Type of Tweet } & Frequency \\
\hline Fellows' Journal Club & 19 \\
Editor's Choice & 11 \\
Tweet Chat & 122 \\
Case & $200^{\mathrm{a}}$ \\
Article & 384 \\
Blog & 159 \\
Other & 137 \\
\hline
\end{tabular}

a 186/200 "Case" tweets had a high engagement rate. $\chi^{2}$ analysis of "Case" (200) versus remaining Tweet types (832) showed a significantly higher engagement rate $(P<.001$; odds ratio, 140.238 [95\% Cl, 77.387-254.133]).

\section{RESULTS}

A total of 1032 tweets were published during the study period. Of these, $405(39.2 \%)$ tweets included an image, and $751(72.8 \%)$ tweets had a hashtag. There were $122(11.8 \%)$ tweets were posted on a weekend. Most tweets were posted in the morning (606; $58.7 \%)$ and afternoon $(318 ; 30.8 \%)$ hours (Table 1$)$. The months of March (106 tweets) and August (100 tweets) had the highest AJNR Twitter activity (Fig 1). Overall, 258/1032 tweets were included in the "high engagement" category (75th percentile). The engagement rate of these tweets was $6.57 \%$ and above. The mean engagement rate of all tweets was $4.75 \%$, and the median engagement rate was $3.4 \%$.

Using the logistic regression model, the presence of an image independently increased the tweet engagement rate by 28.75 times $(P<.001)$, and the presence of a hashtag increased the rate by 3.27 times $(P<.001)$. Tweets published in the morning hours were twice as likely to have a high engagement rate compared with those published in the afternoon hours $(P=.001)$. No significant difference was found in tweets published on weekends compared with weekdays, evening or night compared with morning or afternoon, or according to the month (Table 1). In an independent $\chi^{2}$ analysis, the "Case" tweet type was 140fold more engaging compared with all other tweet types because of the presence of a combination of engaging factors (Table 2). The 10 tweets with the highest engagement rate during the study period are shown in the On-line Appendix.

\section{DISCUSSION}

Our study examined the impact of tweet characteristics on user engagement and demonstrated that tweets including an image or hashtag and those published in the morning hours had the highest engagement rate.

Kelly et $\mathrm{al}^{2}$ showed that AJNR had the second highest number of tweets in the year 2015 (3274) among all radiology journals with an official Twitter presence, second only to the Journal of Vascular and Interventional Radiology. AJNR also had the greatest number of favorites (a form of engagement) per 100 followers. They also showed that radiology journals with Twitter profiles have higher Impact Factors than those without profiles, as well as a positive association between Impact Factor and the number of followers of a journal's Twitter profile. $\operatorname{Cosco}^{3}$ has also shown a strong association of Impact Factors and citations of general medical journals with a journal's Twitter following. Radiology articles published in journals have been disseminated through Twitter and have been shown to be effective in increasing readership. Hoang et $\mathrm{al}^{4}$ showed increased readership of an AJNR article after promotion of the article on social media. Private practice radiology groups are adopting social media platforms (Facebook and Twitter) earlier than academic radiology departments, indicating the use of social media extending beyond research and academics. ${ }^{5}$

Twitter has been used to increase user engagement at various national scientific meetings. Hawkins et $\mathrm{al}^{1}$ showed increased use of Twitter at the 2011 and 2012 Radiological Society of North America (RSNA) annual meetings. The recent annual meeting of 
the American Society of Regional Anesthesia and Pain Medicine showed increased user engagement on Twitter, with Twitter content being scientific and created by meeting attendees, which also engaged nonattendees as evidenced by "retweeting," mostly of picture-containing tweets. ${ }^{6}$ Similarly, the Irish Society of Urology Annual Meeting 2014 demonstrated that Twitter use facilitated interaction between delegates and significant participation of virtual followers. ${ }^{7}$

In the present study, we found that the presence of an image independently increased the engagement rate by nearly 29 times. This may be related to radiology images being more engaging than text tweets. It has been suggested by some Twitter experts to use photos to drive engagement. ${ }^{8}$ We also found that tweets containing a hashtag have a 3-fold increased engagement rate compared with those without a hashtag. A hashtag refers to a word or phrase preceded by a hash or pound sign (\#) and is used to identify messages on a specific topic. The use of hashtags gives more visibility to the tweet because users can search for common topics of interest, such as \#ASNR17, \#RadRes, and \#NeuroRad, which in turn may contribute to increased engagement. Tracking hashtag use over time is possible by using third-party Web sites. Symplur (Upland, California), a health care social media analytics organization, was used by Hawkins et $\mathrm{al}^{1}$ for their study to track the hashtags \#RSNA11 and \#RSNA12. Symplur was also used by Gouda et $\mathrm{al}^{9}$ to track hashtags associated with heart failure.

Our study also analyzed the impact of the time of day on tweet engagement. The highest Twitter activity for @TheAJNR was during the morning hours, which also had twice higher engagement compared with afternoon tweets. No significant difference was found between morning and afternoon tweets compared with evening and night tweets, possibly because of the small number of later-hour tweets.

One limitation of this study was the inclusion of only 1 year of tweets. In addition, the high engagement rate of image-containing tweets may be related to the inherent nature of radiology as an imaging-based medical specialty, and further research is nec- essary to identify the engaging factors for other specialties and organizations.

\section{CONCLUSIONS}

Tweets including an image and/or a hashtag and those published in the morning hours had the highest user engagement rate for the AJNR Twitter feed. Our data may allow other organizations and individual Twitter users to maximize the potential impact of their social media efforts. Further research is required to identify if this high engagement on Twitter leads to meaningful engagement with the journal itself.

\section{REFERENCES}

1. Hawkins CM, Duszak R, Rawson JV. Social media in radiology: early trends in Twitter microblogging at radiology's largest international meeting. J Am Coll Radiol 2014;11:387-90 CrossRef Medline

2. Kelly BS, Redmond CE, Nason GJ, et al. The use of Twitter by radiology journals: an analysis of Twitter activity and Impact Factor. J Am Coll Radiol 2016;13:1391-96 CrossRef Medline

3. Cosco TD. Medical journals, impact and social media: an ecological study of the Twittersphere. CMAJ 2015;187:1353-57 CrossRef Medline

4. Hoang JK, McCall J, Dixon AF, et al. Using social media to share your radiology research: how effective is a blog post? J Am Coll Radiol 2015;12:760-65 CrossRef Medline

5. Glover M, Choy G, Boland GW, et al. Radiology and social media: are private practice radiology groups more social than academic radiology departments? J Am Coll Radiol 2015;12:513-18 CrossRef Medline

6. Schwenk ES, Jaremko KM, Gupta RK, et al. Upgrading a social media strategy to increase Twitter engagement during the spring annual meeting of the American Society of Regional Anesthesia and Pain Medicine. Reg Anesth Pain Med 2017;42:283-88 CrossRef Medline

7. Nason GJ, O'Kelly F, Bouchier-Hayes D, et al. Twitter expands the reach and engagement of a national scientific meeting: the Irish Society of Urology. Ir J Med Sci 2015;184:685-89 CrossRef Medline

8. Stecyk J. \#TweetTip: use photos to drive engagement. https://blog. twitter.com/2015/tweettip-use-photos-to-drive-engagement. Accessed April 2, 2017

9. Gouda P, Das D, Clark A, et al. The impact and implications of Twitter for cardiovascular medicine. J Card Fail 2017;23:266-67 CrossRef Medline 